\title{
Histone Deacetylase
}

National Cancer Institute

\section{Source}

National Cancer Institute. Histone Deacetylase. NCI Thesaurus. Code C16682.

Histone acetylation and deacetylation alternately exposes and occludes DNA to transcription factors. Histone deacetylation is catalyzed by the histone deacetylases.

There are at least 2 classes of HDACs, class I consisting of proteins homologous to yeast Rpd3 (e.g., HDAC1, HDAC2, and HDAC3) and class II consisting of proteins homologous to yeast Hda1 (e.g., HDAC4 and HDAC6). (from OMIM) 